\title{
A case report of mesenteric panniculitis
}

\author{
Ming-En $Z^{1,2}$, Xu-Biao $P^{1,2}$, Cai-Rang $Y^{2}$, Li R ${ }^{2}$, Ling-Qing $Z^{2}$, Zhi-Xin $W^{2}$ and Hing-Ning ${ }^{2,3 *}$ \\ ${ }^{1}$ Medical College of Qinghai University, Xining, China \\ ${ }^{2}$ Department of Hepatopancreatobiliary Surgery, The Affiliated Hospital of Qinghai University, Xining, China \\ ${ }^{3}$ Qinghai Province Key Laboratory of Hydatid Disease Research, Xining, China
}

\begin{abstract}
Mesenteric panniculitis was clinically rare mesenteric chronic inflammatory disease, mesenteric panniculitis diagnosis is difficult and easy to be misdiagnosed. We reported a 65-year-old male patient mainly presenting with abdominal pains mainly through typical CT findings to be confirmed and we used immunosuppressive therapy to cure the patient.
\end{abstract}

\section{Introduction}

Mesenteric panniculitis (MP) was clinically rare mesenteric chronic inflammatory disease, the cause of MP is unknown, and more consideration is due to abdominal surgery, trauma, ischemia, low immunity, drugs, allergies, autoimmune diseases, MP diagnosis is difficult and easy to be misdiagnosed. Unfortunately, there is still no consistent diagnosis and treatment principle, We hope the understanding of the clinical manifestations and auxiliary examination of MP is strengthened through the study of this case.We also reports the process of diagnosis and treatment of MP and the review of MP related literature, especially the mastery of CT imaging features, so can contribute to this diseases diagnosis and treatment.

\section{Case report}

The patient, male, 65 years old, developed intermittent abdominal pain without obvious cause for 2 years, aggravated for 4 days before admission, the pain was moderate degree, tolerable, with dizziness, no fever, no nausea, vomiting and diarrhea. The patient was treated in a local hospital, considering the diagnosis: acute cholecystitis, but the effect was not good. The patient had abdominal pain again 4 days before admission, with pain in the lower back, the pain was heavier than before, and accompanied with occasional vomiting, no fever, no nausea and diarrhea. The abdomen was flat, soft. middle and upper abdominal tenderness positive, rebound tenderness suspicious, Murphys sign negative, and total abdomen did not touch abnormal mass when Just admitted. And there were no abnormalities in the patient's vital signs. In Addition, the patient had surgery for coronary heart disease 2 years ago.

The hematological examination showed: white blood cell count $16.67 \times 10^{9} / \mathrm{L}$, neutrophil percentage $92.6 \%$, neutrophil count $15.44 \times 10^{9} / \mathrm{L}$; urine $\mathrm{pH} 5.0$, urine specific gravity $>1.030$, urea $23.4 \mathrm{mmol} /$ $\mathrm{L}$, creatinine $169 \mathrm{umol} / \mathrm{L}(\mathrm{BUN} / \mathrm{Cr}>40$ ), potassium $5.40 \mathrm{mmol} / \mathrm{L}$; CRP $150.000 \mathrm{mg} / \mathrm{L}$, myocardial enzymes, blood urease amylase, serum tumor markers and immune function indicators were basically normal. Abdominal X-ray film showed incomplete intestinal obstruction; chest, abdomen, pelvic $\mathrm{CT}$ and mesenteric arteriovenous vascular $\mathrm{CT}$ showed: 1. pulmonary infection; 2 . mild expansion of the lower common bile duct, choledocholithiasis? 3 . The mesenteric part of the middle part of the abdomen is spiral, the surrounding exudation, the surrounding adenopathy show; 4 . The pancreas is normal; 5 . Prostatic hyperplasia, a small amount of pelvic fluid; And there was no thrombosis in the superior mesenteric arteriovenous and portal vein (Figure 1).

Combined with the patient's clinical symptoms, past history, we consider the main diagnosis were: choledocholithiasis, biliary infection, pulmonary infection, incomplete intestinal obstruction, so we let the

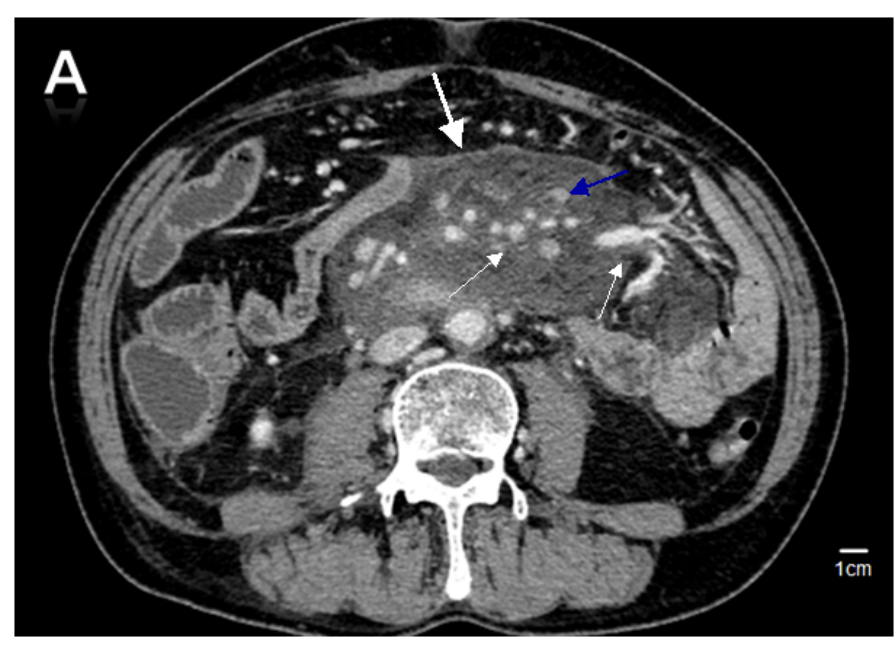

Figure 1. Image from CT scan of abdomen performed with intravenous contrast Materia: the mesenteric part of the middle part of the abdomen is spiral, the surrounding adenopathy show, (blue arrow), encases branches of the superior mesenteric artery and tributaries of the superior mesenteric vein (thin white arrow) "wrap" around the mesentery("pseudocapsule sign")(thicken white arrows)

${ }^{*}$ Correspondence to: Hai-Ning Fan, Department of Hepatopancreatobiliary Surgery, the Affiliated Hospital of Qinghai University, China, E-mail: fanhaining8686@163.com

Key words: Mesenteric panniculitis, Diagnosis, Treatment, Immunosuppressive therapy

Received: November 01, 2018; Accepted: November 12, 2018; Published: November 14, 2018 
patient didn't eat, but could drink a small amount of water, and given him anti-infection treatment, dilatancy and fluid infusion, expansion of coronary vessels, antispasmodic, laxative and other symptomatic treatment. And two stones in bile duct were taken out after performed with ERCP (Figure 2).

However, the patient's abdominal pain was not relieved, but intermittently aggravated, and fever was performed twice during the period, the highest of the degree was to $38.5^{\circ} \mathrm{C}$. Physical examination revealed that the abdomen was slightly larger than early, and the palpation of the abdomen was slightly tense, the upper abdomen was tender with rebound tenderness. And then CT display of abdominal cavity showed: the mesenteric part of the middle part of the abdomen is spiral, the mesangial thickening, the surrounding exudation more aggravated than before, the surrounding adenopathy show and formation of typical "pseudocapsule sign", the diagnosis of mesenteric panniculitis is considered, and the tumor may be excluded (Figure 3). According to the patient's symptoms, signs, typical imaging characteristics, and the advice of doctor of rheumatism and immunology. the diagnosis was mesenteric panniculitis. And we added $80 \mathrm{mg}$ of methylprednisolone to him one day and changed the dose to $40 \mathrm{mg}$ after 3 days. the symptoms of abdominal pain, vomiting, hiccups and other symptoms were significantly relieved, and the diet and sleep were significantly improved after 6 days of using methylprednisolone, the blood picture was reduced to normal, the liver and kidney function were normal, and the examination was not special. Review of abdominal CT scan at discharge (Figure 4).

\section{Discussion}

Mesenteric panniculitis (MP), also known as sclerosing mesenteric inflammation, mesenteric lipodystrophy, intestinal fat metabolism disorder, mesenteric Weber-Christian disease, etc., was originally described by Ogden and clinically rare mesenteric chronic inflammatory disease [1-3]. The author searches for MP related literature and discusses it in conjunction with this case.

Etiology: The cause of MP is unknown, and more consideration is due to abdominal surgery, trauma, ischemia, low immunity, drugs, allergies, autoimmune diseases, etc. [1-4]. This patient has no history of abdominal trauma or surgery, no history of taking special drugs, and the autoimmune-related indicators are basically normal. we guessed

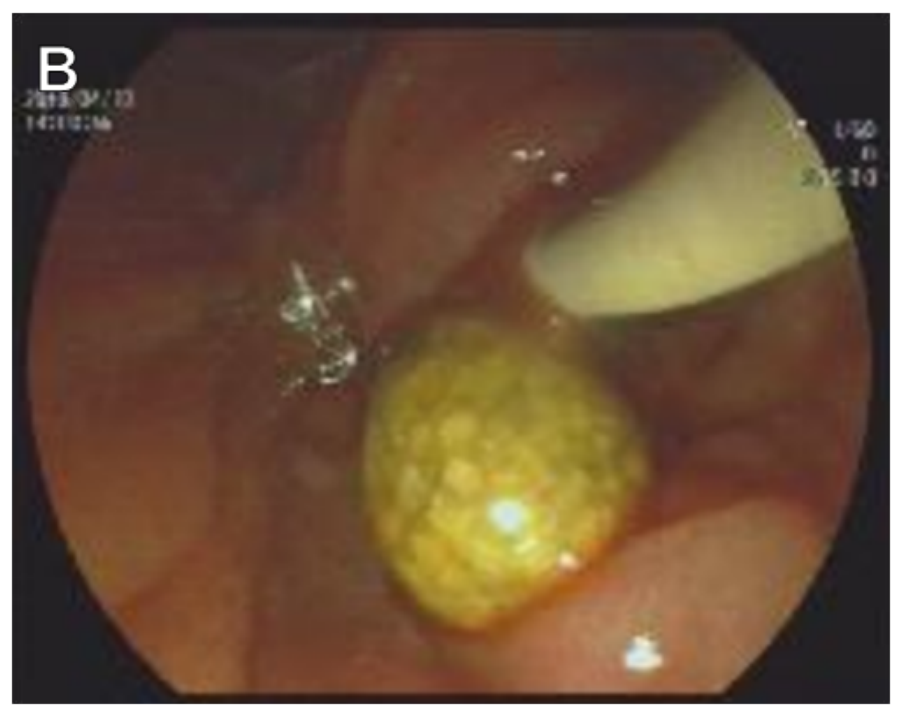

Figure 2. The stones in bile duct were taken out after performed with ERCP

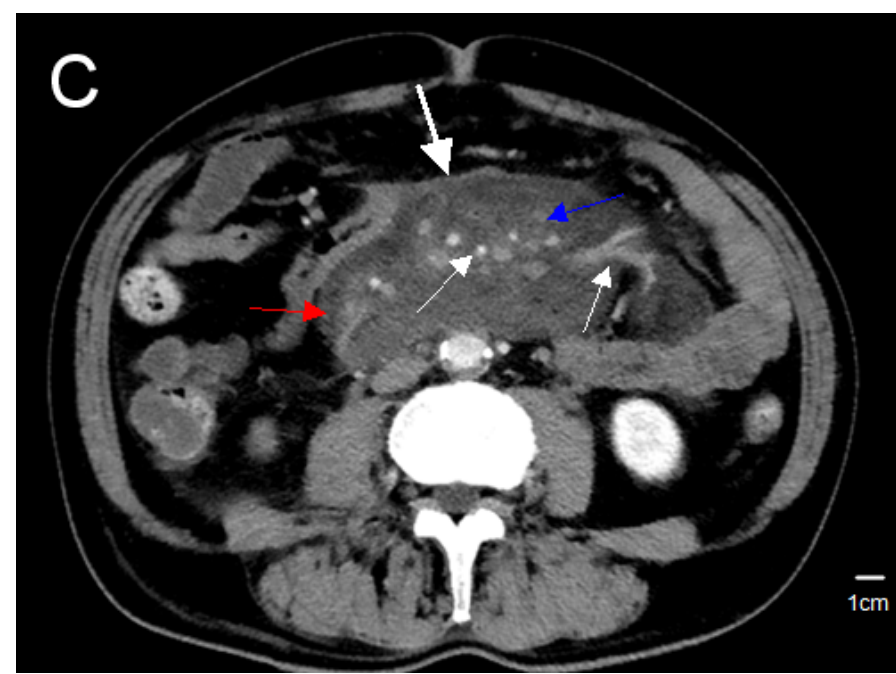

Figure 3. Image from CT scan of abdomen performed with intravenous contrast Materia: A central mesenteric soft-tissue mass $(11.6 \times 6.8 \mathrm{~cm})$, the mesangial thickens, the surrounding exudation was a litter more than before(red arrows), the surrounding adenopathy show, ( blue arrow), encases branches of the superior mesenteric artery and tributaries of the superior mesenteric vein(thin white arrow), "wrap" around the mesentery("pseudocapsule sign")thicken white arrows)

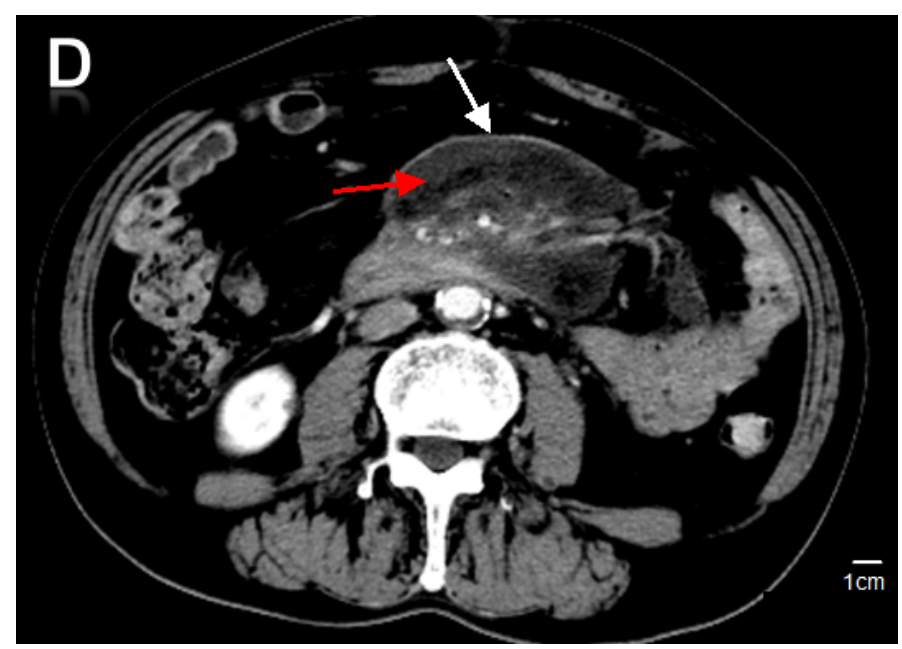

Figure 4. Image from CT scan of abdomen performed with intravenous contrast Materia: the spiral and exudation of mesenteric were better than before (thin arrows), and reduction in the size of the mesenteric soft-tissue mass $(6.8 \times 5.0 \mathrm{~cm})$, "pseudocapsule sign" was still there (red arrows)

may be related to the patient's physical weakness and the history of cardiac surgery.

\section{Clinical features}

The disease can occur in any age group, more common in 50 to 60 years old, male to female ratio of 1.5-1.8:1.0. MP patients have different clinical manifestations, usually bloating, nausea, vomiting, fever, weight loss, loss of appetite, weight loss, etc. Some patients may also have no special discomfort. A few patients present with abdominal mass, peritonitis, peritoneal irritation, ascites, etc., [1-3]. In this case, the abdominal pain was obvious when admitted to the hospital. It was easy to consider the cholangitis caused by the choledocholithiasis. The diagnosis of mesenteric panniculitis was ignored. The abdominal distention of the patients was not relieved 3 days after ERCP stone removal, and vomiting occurred again. Combined with abdominal CT results, we consider MP combined with choledocholithiasis. 


\section{Diagnosis}

At present, there is no consistent diagnostic standard for MP. At present, there are more data showing the increase of inflammatoryrelated indicators such as white blood cells, CRP and erythrocyte sedimentation rate in laboratory tests, but it is necessary to point out that the specific laboratory has not yet been determined. test result. Most patients have no abnormalities in biochemical tests, urine routines, and stool routines. When the patient was admitted to the hospital, the white blood cells and CRP were elevated. The biochemical routine liver function and renal function were abnormal. The inflammatory reaction may be related to the MP itself. It is not excluded from the pulmonary infection. The renal function abnormality is considered to be insufficient for the whole-body fluid capacity.and the renal function gradually returned to normal after symptomatic supportive treatment with rehydration.

In addition, contrast-enhanced CT seems to be more important for the diagnosis of MP, While MP was usually found by B-ultrasound. B-ultrasound can show mesenteric space occupying, but it is not sure of placeholder nature. CT examination is the preferred method for diagnosing this disease. Typical cases can be directly diagnosed without pathological biopsy. The typical manifestations of MP abdominal CT are single or multiple fat-based masses with mesenteric vessels surrounding the mesangial vessels, uneven density, and clear boundaries.MP exhibit a certain degree of inflammatory response and fibrosis, and the lesions are visible around the lesions. A fatty halo, a small number of cystic masses (lymphatic vessels due to lymphatic vessels and vascular obstruction $)^{[1,2]}$. This patient's CT display of abdominal cavity showed that local mesenteric thickening, swirling, and peripheral exudation in the middle part of the abdomen, and fat and blood vessel encapsulation changes, the scattered lymph nodes was in surround (Figure 2). It meets the typical CT findings of MP and is highly suspected of mesenteric panniculitis. The MRI performance of MP is not specific, but it is clearer about fat, soft tissue components and vascular involvement. The patient's CT was highly suspected of being MP, and there was no MRI Because of patient's heart stent.

However, these findings are not MP-specific, and previous reports have shown that MP patients are sometimes misdiagnosed as Crohn's disease. For the diagnosis of MP, pathophysiology and its specific markers are increasingly needed. If the pathological tissue is available, the mesentery of the lower eye is massive, the rubbery sample becomes thick, and the hard tissue is scarred, and the scar of the fibrous tissue shrinks, causing the mesentery to shorten and form a mass, and the intestinal ducts adhere to each other to form an angle. There are scattered irregular grayish yellow, reddish brown fat necrosis in the lesion. Many foamy lipophilic cells and macrophages infiltrated can be seen under the microscope, and there were different degrees of fibrosis, fat necrosis and calcification under the microscope, which can diagnose MP [1-3].
Pathology is the gold standard for the diagnosis of this disease, but it is difficult to obtain mesenteric tissue specimens. Not all patients need to obtain histological diagnosis.

\section{Treatment and prognosis}

MP are a benign inflammatory lesion with self-limiting, MP has good prognosis, few recurrences after healing, and few serious complications [1,3-5]. Because the disease causes extensive or localized mesenteric inflammatory changes, the location is close to the larger blood vessels, and it is prone to recurrence after local ablation. If there is no special clinical, surgical treatment is not recommended in principle. When MP is complicated with intestinal obstruction, peritonitis and other serious complications. Intestinal resection, necrosis removal, and intestinal adhesion lysis can be considered for the tumor or corresponding lesion. According to the specific conditions of patients, conservative treatment can be used for anti-infective and/or immunosuppressive treatment, and glucocorticoids, non-steroidal anti-inflammatory drugs, colchicine, progesterone and cyclophosphamide are selected, but the efficacy reports are different $[2,3,5]$. This patient was not treated with surgery. He was treated with methylprednisolone alone. The symptoms were relieved obviously, and the effect was significant, but the follow-up long-term follow-up was needed to observe the effect after treatment.

\section{Conflicts of interest}

\section{None}

\section{Acknowledgments}

The authors thank all who helped during the writing of this thesis.

\section{Author Contribution}

MEZ and XBP: Involved in patient management and wrote the manuscript. LQZ and LR: Involved in patient management. ZXW and DYCR: Processed data and images; HNF: provided editing and review of the manuscript.

\section{References}

1. Akram S, Pardi DS, Schaffner JA (2007) Sclerosing mesenteritis: clinicalfeatures, treatment, and outcome in ninety-two patients. Clin Gastroenterol Hepatol 5: 523-524. [Crossref]

2. Sharma P, Yadav S, Needham CM (2017) Sclerosing mesenteritis: a systematic review of 192 cases. Clin J Gastroenterol 10: 103-111. [Crossref]

3. Shinta M, Masatoshi W, Yujiro M (2017) Increased levels of prostaglandin E-major urinary metabolite (PGE-MUM) in active mesenteric panniculitis patients. Medicine 96: e9237. [Crossref]

4. Hussein MR, Abdelwahed SR (2015) Mesenteric panniculitis: an update. Expert Rev Gastroenterol Hepatol 9: 67-78. [Crossref]

5. Coulier B (2011) Mesenteric panniculitis. Part 2: prevalence and natural course: MDCT prospective study. JBR-BTR 91: 241-246. [Crossref]

Copyright: ( 2018 Ming-En Z. This is an open-access article distributed under the terms of the Creative Commons Attribution License, which permits unrestricted use, distribution, and reproduction in any medium, provided the original author and source are credited. 